\title{
Determine Water Quality of Barapukuria Coal Mine Company Ltd and Its Surrounding Area in Dinajpur District
}

\author{
Md. Reazul Islam, Md. Moshfequr Rahman \\ Geological Survey of Bangladesh, Segunbagicha, Dhaka, Bangladesh
}

Email address:

reazul2005@gmail.com (Md. R. Islam)

\section{To cite this article:}

Md. Reazul Islam, Md. Moshfequr Rahman. Determine Water Quality of Barapukuria Coal Mine Company Ltd and Its Surrounding Area in Dinajpur District. Journal of Water Resources and Ocean Science. Vol. 10, No. 1, 2021, pp. 9-15. doi: 10.11648/j.wros.20211001.12

Received: January 15, 2021; Accepted: February 6, 2021; Published: April 16, 2021

\begin{abstract}
The purpose of this programme is to determine water quality of Barapukuria Coal Mining Company Ltd. and its surrounding area in Dinajpur district. 36 samples of water (Coal water flow stream drain water, surrounding area Water samples of water from the surface of the surface and tube type of tube well) are collected from this study area. Current $\mathrm{pH}$, Conductivity, TDS, DO, temperature Samples physical character are measured and recorded in notebooks. After marking it properly, by adding $\mathrm{HNO}_{3}$ control the collected samples $\mathrm{pH} 500 \mathrm{ml}$ Polypropylene bottles are stored in ice box samples for chemical laboratories. Besides, the information related to the impact on the area is collected by the Barapukuria Coal Mining Company Ltd. and Barapukuria Coal-based thermal power plant. The amount of $\mathrm{Al}, \mathrm{Fe}, \mathrm{Ti}, \mathrm{Cu}, \mathrm{Co}, \mathrm{Cd}, \mathrm{Zn}, \mathrm{Ca}, \mathrm{Mn}, \mathrm{Mg}, \mathrm{Na}$, $\mathrm{K}$, As of the samples collected from the Analytical Chemistry Laboratory of Geological Survey of Bangladesh was determined. Chemical analysis of the samples showed that excessive presence of any element was not exist ECR (Environment Conservation Rule). 1997 of Bangladesh Standard value.
\end{abstract}

Keywords: Barapukuria, pH, Plant, Chemicals

\section{Introduction}

\subsection{Purpose and Scope of the Work}

Barapukuria coal mine \& Barapukuria thermal power plant are situated at Parbotipur upazilla in Dinajpur District, Bangladesh. Area of Barapukuria coal mine is 646 acres of agricultural plain land. Barapukuria thermal power is established of 245 acres land. Coal, a natural mineral resource, is a black or brownish-black rock that is formed from plants, which died about 100 to 400 million years ago [1]. It is a heterogeneous mixture of several components such as sulfur, elemental carbon, arsenic, ash and heavy metals etc [2]. Coal has been a major means of fuel used in electricity generation. It has played a huge role in the development drive of several nations. Industrial revolutions had been powered by coal and the energy it supplied. In many countries coal is the primary source of energy [3, 4$]$.

Coal is the second largest stock of energy in Bangladesh. 41.5 percent electricity in the world is produced from coal [7]. In order to alleviate the electricity crisis of Bangladesh, Barapukuria Coal and Power project is a blessing indeed.
Estimated energy from the project is equivalent to 53 trillion cubic feet (TCF) of natural gas; more than threefold of gas reserves of the country. So, a new dimension is added to the economy of Bangladesh, regarded as coal mine industry. Bangladesh is one of the top coal production countries and supply coal for its internal industrial energy source [13]. Barapukuria coal mine has the capacity to extract 3,500-4,500 tons coal per day. Among them 3,500 tons is used Barapukuria thermal power plant for electric power generation [6].

Assessing the coal mine and its versatile impact over the industrial revolution time, the researcher, end of the 20th century revealed that there is huge risk of health, potential air pollution, noticeable change in landscape, political and social problem, overall sustainability of the environment could get seriously affected by coal mine operation [12]. During the mining process huge amounts of water are discharged on the surface, which often contains high loads of TSS, TDS, hardness and heavy metals and consequently contaminates the surface and groundwater [5]. Therefore, it is obvious that an assessment of the local environment should go prior and along the project of Barapukuria before any unexpected consequence overwhelms this project. In order to find out 
possible positive and negative benefits, a thorough analysis considering all the impacts on soil, water, sound levels, and changes in aesthetic environment, were carried out in this study $[15,16]$. The major objectives of the study were (i) to assess the socioeconomic impacts of these projects, (ii) to assess the impacts on land use pattern and (iii) to identify and quantify the environmental effects.

Coal is washed before reaching the thermal power plant. This coal washed water is drained into near paddy filed, pond, canal and river. Thus the areas of Barapukuria coal mine are severely contaminated by coal waste water [11]. In spite of the role coal plays, there are strong evidences of coal's impact on human health and the environment during every stage of its mining, use, and post-combustion disposal [14]. Air pollution generated by coal mining and combustion in power plants [8].
This programme aims is to identify the influence of mine and thermal power plants on the adjoining areas.

\subsection{Location of the Study Area}

The present study area was carried out to observe environmental effects from Barapukuria coal mining industries and Barapukuria thermal power plant in Dinajpur, Bangladesh. Barapukuria coal mine and power plant site is located in flat paddy land of the north-western corner of Bangladesh at about $45 \mathrm{~km}$ east of the district headquarters of Dinajpur, $20 \mathrm{~km}$ east from the border of India. The geographical location of the studied area is in between $25.6533^{\circ} \mathrm{N}$ to $25.6533^{\circ} \mathrm{N} \&$ $88.9155^{\circ} \mathrm{E}$ to $88.9155^{\circ} \mathrm{E}$. Location of the working area was noted in terms of longitude and latitude by using GPS.

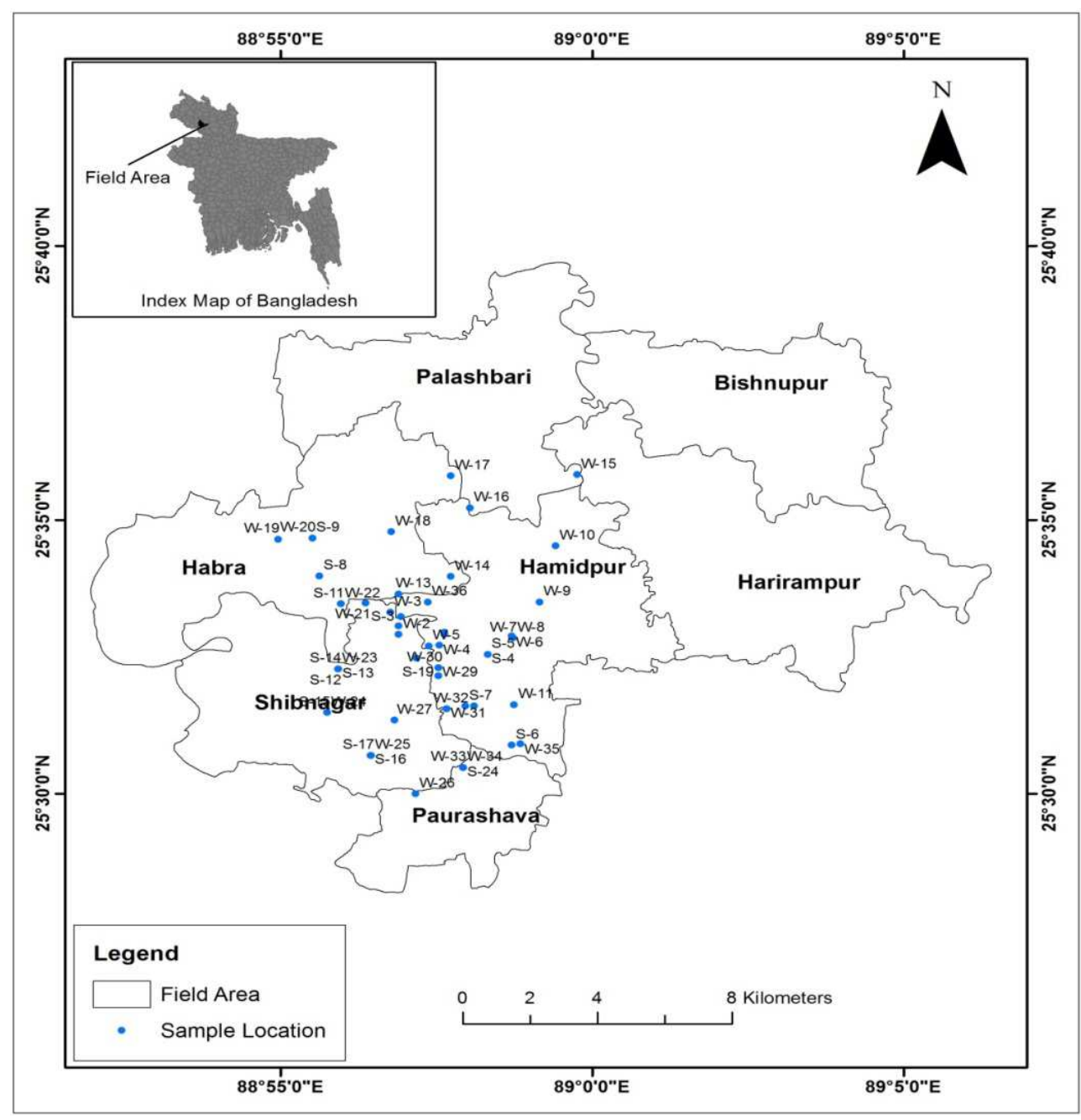

Figure 1. Location of the study area and water sample sampling point.

\subsection{Previous Work}

Different research and studies have been done by different scientists throughout the world including our country about the impact on the environment of Baropukuria coal mine industry \& Barapukuria thermal power plant. Geological Survey of Bangladesh has not done yet any complete relevant study.

\section{Methodology}

\subsection{Sample Collection}

Assessment the effect of Barapukuria coal mine and thermal power plant on surrounding environment, the effect of coal water on soil, water, coal waste water and emissions 
from power plant had been evaluated. The study was based on field observations, sample collections and testing. Soil and drain water samples were collected from the study area for sampling.

Table 1. Description of location of collected water samples.

\begin{tabular}{|c|c|c|c|c|c|c|c|c|}
\hline $\begin{array}{l}\text { Sl } \\
\text { No }\end{array}$ & $\begin{array}{l}\text { Sample } \\
\text { No }\end{array}$ & Sample source & Village & Post Office & $\begin{array}{l}\text { Upazilla } \\
\text { \& District }\end{array}$ & Latitude & Longitude & Depth \\
\hline 1 & RMW-1 & Baropukuria coal mine ETP outlet water & Chowhati & Baropukuria & \multirow{33}{*}{$\begin{array}{l}\text { Parbotipur, } \\
\text { Dinajpur }\end{array}$} & $25^{\circ} 32^{\prime} 55^{\prime \prime} \mathrm{N}$ & $88^{\circ} 57^{\prime} 38^{\prime \prime} \mathrm{E}$ & Surface \\
\hline 2 & RMW-2 & $\begin{array}{l}\text { Baropukuria Thermal Power Plant Ash dump } \\
\text { pond area }\end{array}$ & Durgapur & Baropukuria & & $25^{\circ} 33^{\prime} 04^{\prime \prime} \mathrm{N}$ & $88^{\circ} 56^{\prime} 54^{\prime \prime} \mathrm{E}$ & Surface \\
\hline 3 & RMW-3 & $\begin{array}{l}\text { Baropukuria Thermal Power Plant drainage } \\
\text { outlet }\end{array}$ & Durgapur & Baropukuria & & $25^{\circ} 33^{\prime} 19^{\prime \prime} \mathrm{N}$ & $88^{\circ} 56^{\prime} 46^{\prime \prime} \mathrm{E}$ & Surface \\
\hline 4 & RMW-4 & $\begin{array}{l}\text { Baropukuria coal mine Gondoana layer water } \\
\text { outlet }\end{array}$ & Zigagari & Baropukuria & & $25^{\circ} 32^{\prime} 43^{\prime \prime} \mathrm{N}$ & $88^{\circ} 57^{\prime} 33^{\prime \prime} \mathrm{E}$ & Surface \\
\hline 5 & RMW-5 & $\begin{array}{l}\text { Baropukuria coal mine Gondoana layer water } \\
\text { outlet end point }\end{array}$ & - & Baropukuria & & $25^{\circ} 32^{\prime} 42^{\prime \prime} \mathrm{N}$ & $88^{\circ} 57^{\prime} 23^{\prime \prime} \mathrm{E}$ & Surface \\
\hline 6 & RMW-6 & Tube-well water & Bashpukur & Baropukuria & & $25^{\circ} 32^{\prime} 53^{\prime \prime} \mathrm{N}$ & $88^{\circ} 58^{\prime} 43^{\prime \prime} \mathrm{E}$ & $30 \mathrm{~m}$ \\
\hline 7 & RMW-7 & Tube-well water & Bashpukur & Baropukuria & & $25^{\circ} 32^{\prime} 51^{\prime \prime} \mathrm{N}$ & $88^{\circ} 58^{\prime} 44^{\prime \prime} \mathrm{E}$ & $30 \mathrm{~m}$ \\
\hline 8 & RMW-8 & Pond Water & Bashpukur & Baropukuria & & $25^{\circ} 32^{\prime} 51^{\prime \prime} \mathrm{N}$ & $88^{\circ} 58^{\prime} 44^{\prime \prime} \mathrm{E}$ & Surface \\
\hline 9 & RMW-9 & Tube-well water & Dhulaodal & Baropukuria & & $25^{\circ} 33^{\prime} 30^{\prime \prime} \mathrm{N}$ & $88^{\circ} 59^{\prime} 09^{\prime \prime} \mathrm{E}$ & $30 \mathrm{~m}$ \\
\hline 10 & RMW-10 & Tube-well water & Khalilpur & Khaierpur & & $25^{\circ} 34^{\prime} 32^{\prime \prime} \mathrm{N}$ & $88^{\circ} 59^{\prime} 25^{\prime \prime} \mathrm{E}$ & $33 \mathrm{~m}$ \\
\hline 11 & RMW-11 & Tube-well water & Pachghoria & Baropukuria & & $25^{\circ} 31^{\prime} 38^{\prime \prime} \mathrm{N}$ & $88^{\circ} 58^{\prime} 45^{\prime \prime} \mathrm{E}$ & $30 \mathrm{~m}$ \\
\hline 13 & RMW-13 & Tube-well water & Eusufpur & Shohidpur Hat & & $25^{\circ} 33^{\prime} 39^{\prime \prime} \mathrm{N}$ & $88^{\circ} 56^{\prime} 54^{\prime \prime} \mathrm{E}$ & $28 \mathrm{~m}$ \\
\hline 14 & RMW-14 & Tube-well water & East Eusufpur & Shohidpur Hat & & $25^{\circ} 33^{\prime} 58^{\prime \prime} \mathrm{N}$ & $88^{\circ} 57^{\prime} 44^{\prime \prime} \mathrm{E}$ & $30 \mathrm{~m}$ \\
\hline 15 & RMW-15 & Tube-well water & Hossainpur & Khaierpukur & & $25^{\circ} 35^{\prime} 50^{\prime \prime} \mathrm{N}$ & $88^{\circ} 59^{\prime} 46^{\prime \prime} \mathrm{E}$ & $36 \mathrm{~m}$ \\
\hline 16 & RMW-16 & Tube-well water & Kalikapur & Shohidpur Hat & & $25^{\circ} 35^{\prime} 13^{\prime \prime} \mathrm{N}$ & $88^{\circ} 58^{\prime} 03^{\prime \prime} \mathrm{E}$ & $30 \mathrm{~m}$ \\
\hline 17 & RMW-17 & Tube-well water & Khamarpara & Durgapur & & $25^{\circ} 35^{\prime} 48^{\prime \prime} \mathrm{N}$ & $88^{\circ} 57^{\prime} 44^{\prime \prime} \mathrm{E}$ & $35 \mathrm{~m}$ \\
\hline 18 & RMW-18 & Tube-well water & Bhabanipur Bazar & Bhabanipur & & $25^{\circ} 34^{\prime} 47^{\prime \prime} \mathrm{N}$ & $88^{\circ} 56^{\prime} 47^{\prime \prime} \mathrm{E}$ & $32 \mathrm{~m}$ \\
\hline 19 & RMW-19 & Tube-well water & Chowhali & Habra & & $25^{\circ} 34^{\prime} 39^{\prime \prime} \mathrm{N}$ & $88^{\circ} 54^{\prime} 58^{\prime \prime} \mathrm{E}$ & $38 \mathrm{~m}$ \\
\hline 20 & RMW-20 & River water & Chowhali & Habra & & $25^{\circ} 34^{\prime} 40^{\prime \prime} \mathrm{N}$ & $88^{\circ} 55^{\prime} 31^{\prime \prime} \mathrm{E}$ & Surface \\
\hline 21 & RMW-21 & Thermal plant drainage water & Dhutipukur & Hagirdanga & & $25^{\circ} 33^{\prime} 29^{\prime \prime} \mathrm{N}$ & $88^{\circ} 56^{\prime} 22^{\prime \prime} \mathrm{E}$ & Surface \\
\hline 22 & RMW-22 & Thermal plant drainage outlet end point water & Dhutipukur & Hagirdanga & & $25^{\circ} 33^{\prime} 28^{\prime \prime} \mathrm{N}$ & $88^{\circ} 55^{\prime} 58^{\prime \prime} \mathrm{E}$ & Surface \\
\hline 23 & RMW-23 & River Water & Ramvadrapur & Rajarampur & & $25^{\circ} 32^{\prime} 17^{\prime \prime} \mathrm{N}$ & $88^{\circ} 55^{\prime} 56^{\prime \prime} \mathrm{E}$ & Surface \\
\hline 24 & RMW-24 & River Water & East Jaforepur & Rajarampur & & $25^{\circ} 31^{\prime} 29^{\prime \prime} \mathrm{N}$ & $88^{\circ} 55^{\prime} 45^{\prime \prime} \mathrm{E}$ & Surface \\
\hline 25 & RMW-25 & River Water & Ghatpara & Rajarampur & & $25^{\circ} 30^{\prime} 42^{\prime \prime} \mathrm{N}$ & $88^{\circ} 56^{\prime} 27^{\prime \prime} \mathrm{E}$ & Surface \\
\hline 26 & RMW-26 & River Water & Phulbari & Phulbari & & $25^{\circ} 30^{\prime} 00^{\prime \prime} \mathrm{N}$ & $88^{\circ} 57^{\prime} 10^{\prime \prime} \mathrm{E}$ & Surface \\
\hline 27 & RMW-27 & Tube-well water & Sultanpur & Rajarampur & & $25^{\circ} 31^{\prime} 21^{\prime \prime} \mathrm{N}$ & $88^{\circ} 56^{\prime} 50^{\prime \prime} \mathrm{E}$ & $32 \mathrm{~m}$ \\
\hline 30 & RMW-30 & Tube-well water & Kalupara & Barapukuria & & $25^{\circ} 32^{\prime} 09^{\prime \prime} \mathrm{N}$ & $88^{\circ} 57^{\prime} 32^{\prime \prime} \mathrm{E}$ & $35 \mathrm{~m}$ \\
\hline 31 & RMW-31 & Coal mine drainage water & South Rasulpur & Barapukuria & & $25^{\circ} 31^{\prime} 33^{\prime \prime} \mathrm{N}$ & $88^{\circ} 57^{\prime} 40^{\prime \prime} \mathrm{E}$ & Surface \\
\hline 32 & RMW-32 & Tube-well water & South Rasulpur & Barapukuria & & $25^{\circ} 31^{\prime} 33^{\prime \prime} \mathrm{N}$ & $88^{\circ} 57^{\prime} 40^{\prime \prime} \mathrm{E}$ & $35 \mathrm{~m}$ \\
\hline 33 & RMW-30 & Coal mine drainage water & Mubarakpur & Barapukuria & & $25^{\circ} 30^{\prime} 29^{\prime \prime} \mathrm{N}$ & $88^{\circ} 57^{\prime} 56^{\prime \prime} \mathrm{E}$ & Surface \\
\hline 34 & RMW-30 & Tube-well water & Mubarakpur & Barapukuria & & $25^{\circ} 30^{\prime} 29^{\prime \prime} \mathrm{N}$ & $88^{\circ} 57^{\prime} 56^{\prime \prime} \mathrm{E}$ & $36 \mathrm{~m}$ \\
\hline 35 & RMW-35 & Tube-well water & Chak Moheshpur & Barapukuria & & $25^{\circ} 30^{\prime} 55^{\prime \prime} \mathrm{N}$ & $88^{\circ} 58^{\prime} 51^{\prime \prime} \mathrm{E}$ & $32 \mathrm{~m}$ \\
\hline 36 & RMW-36 & Tube-well water & $\begin{array}{l}\text { Barapukuria Coal } \\
\text { mine area }\end{array}$ & Barapukuria & & $25^{\circ} 33^{\prime} 30^{\prime \prime} \mathrm{N}$ & $88^{\circ} 57^{\prime} 22^{\prime \prime} \mathrm{E}$ & $36 \mathrm{~m}$ \\
\hline
\end{tabular}

For water samples some physical parameter such as Temperature, $\mathrm{pH}$, Dissolved Oxygen (DO) and Conductivity were also carried out in the field (Table 2). Finally, Collected samples were selected according to priority of location for chemical analysis in the Analytical Chemical laboratory of GSB.

Table 2. Description of physical parameter of collected water samples.

\begin{tabular}{llllll}
\hline SI No & Sample No & Temperature $\left({ }^{\circ} \mathbf{C}\right)$ & TDS $(\mathbf{p p m})$ & DO (mg/l) & Conductivity $(\boldsymbol{\mu s})$ \\
\hline 1 & RMW-1 & 38.6 & 124 & 4.51 & 176 \\
2 & RMW-2 & 33.1 & 86.9 & 5.32 & 174.6 \\
3 & RMW-3 & 46 & 41 & 5.2 & 114.5 \\
4 & RMW-4 & 34.2 & 56.8 & 5.51 & 110 \\
5 & RMW-5 & 32.4 & 98.3 & 5.07 & 198.7 \\
6 & RMW-6 & 29.3 & 78.0 & 8.03 & 155.4 \\
7 & RMW-7 & 27.9 & 46.8 & 8.51 & 93.9 \\
8 & RMW-8 & 29 & 78.4 & 9.40 & 7.9 \\
9 & RMW-9 & 27.2 & 41.0 & 8.46 & 7.9 \\
10 & RMW-10 & 27.6 & 45.5 & 8.56 & 7.9 \\
11 & RMW-10 & 26.8 & 58.1 & 9.86 & 7.9 \\
12 & RMW-12 & 28.1 & 63.3 & 9.32 & 7.8 \\
\hline
\end{tabular}




\begin{tabular}{|c|c|c|c|c|c|c|}
\hline SI No & Sample No & Temperature $\left({ }^{\circ} \mathrm{C}\right)$ & TDS (ppm) & DO (mg/l) & Conductivity ( $\mu \mathrm{s})$ & pH \\
\hline 13 & RMW-13 & 27.1 & 45.5 & 5.77 & 91.9 & 7.18 \\
\hline 14 & RMW-14 & 28.6 & 68.9 & 8.46 & 112.8 & 7.46 \\
\hline 15 & RMW-15 & 28.8 & 60.6 & 5.45 & 66.8 & 7.34 \\
\hline 16 & RMW-16 & 27.6 & 69.6 & 7.76 & 139.1 & 6.97 \\
\hline 17 & RMW-17 & 27.9 & 77.4 & 7.32 & 153.2 & 7.34 \\
\hline 18 & RMW-18 & 27.2 & 56 & 8.78 & 92.1 & 7.44 \\
\hline 19 & RMW-19 & 28.3 & 71.6 & 9.26 & 143.2 & 7.42 \\
\hline 20 & RMW-20 & 29.0 & 68.2 & 8.36 & 136.8 & 7.42 \\
\hline 21 & RMW-21 & 33.6 & 128 & 4.01 & 257 & 7.87 \\
\hline 22 & RMW-22 & 30.3 & 128 & 4.02 & 256 & 7.83 \\
\hline 23 & RMW-23 & 27.3 & 127 & 5.32 & 252 & 7.57 \\
\hline 24 & RMW-24 & 27.2 & 126 & 5.86 & 252 & 7.67 \\
\hline 25 & RMW-25 & 28.8 & 127 & 5.92 & 256 & 7.65 \\
\hline 26 & RMW-26 & 29.4 & 120 & 6.32 & 242 & 7.26 \\
\hline 27 & RMW-27 & 26.9 & 53.2 & 8.57 & 106.3 & 7.23 \\
\hline 28 & RMW-28 & 26.8 & 66.1 & 8.56 & 131.7 & 7.50 \\
\hline 29 & RMW-29 & 31.9 & 68.2 & 6.22 & 196.4 & 8.21 \\
\hline 30 & RMW-30 & 28.88 & 76.3 & 8.78 & 149.7 & 7.51 \\
\hline 31 & RMW-31 & 31.6 & 98.1 & 6.32 & 232 & 8.18 \\
\hline 32 & RMW-32 & 27.5 & 83.1 & 8.32 & 164.3 & 7.66 \\
\hline 33 & RMW-33 & 32.1 & 97.7 & 6.97 & 230 & 8.11 \\
\hline 34 & RMW-34 & 27.2 & 86.7 & 8.98 & 172 & 7.61 \\
\hline 35 & RMW-35 & 27.4 & 56.4 & 8.96 & 87.6 & 7.58 \\
\hline 36 & RMW-36 & 27.4 & 67.5 & 8.98 & 136.0 & 7.46 \\
\hline
\end{tabular}

\subsection{Preservation of Water Sample}

Water sample were preserved at $\mathrm{P}^{\mathrm{H}} 1.5$ to 2.0 with addition of Nitric acid just after collection in the field. Samples were stored in individual polypropylene bottles at room temperature with proper identification mark.

\subsection{Chemical Analysis of Water Sample}

The heavy metal ion Lead, Copper, Zinc, Cobalt, Nickel, Cadmium were determined with the help ICP-OES using their selective wave length. The concentration measured as ppm level.

Table 3. Chemical Analysis of water samples.

\begin{tabular}{|c|c|c|c|c|c|c|c|c|}
\hline Sl. No & Sample no & Cd (ppm) & Co (ppm) & $\mathrm{Cu}(\mathrm{ppm})$ & Mn (ppm) & $\mathrm{Ni}(\mathrm{ppm})$ & $\mathrm{Pb}$ (ppm) & Zn (ppm) \\
\hline 1 & RMW-1 & 0.000599 & 0.00546 & 0.01012 & 0.1395 & 0.0292 & 0.0263 & 0.1672 \\
\hline 2 & RMW-2 & 0.00431 & 0.02133 & 0.2939 & 0.5741 & 0.0966 & 0.1261 & 0.1576 \\
\hline 3 & RMW-3 & 0.001836 & 0.00529 & 0.0132 & 0.1013 & 0.0316 & 0.0204 & 0.0532 \\
\hline 4 & RMW-4 & 0.00248 & 0.00762 & 0.01035 & 0.1725 & 0.0216 & 0.0248 & 0.047 \\
\hline 5 & RMW-5 & 0.001138 & 0.00401 & 0.01314 & 0.2058 & 0.0306 & 0.0300 & 0.0681 \\
\hline 6 & RMW-6 & 0.002429 & 0.00587 & 0.01269 & 0.0766 & 0.0221 & 0.0236 & 0.0600 \\
\hline 7 & RMW-7 & 0.001789 & 0.00320 & 0.01399 & 0.2656 & 0.01843 & 0.0274 & 0.13044 \\
\hline 8 & RMW-8 & 0.001456 & 0.00643 & 0.01623 & 0.7440 & 0.01260 & 0.0239 & 0.05353 \\
\hline 9 & RMW-9 & 0.00208 & 0.00537 & 0.00978 & 0.06836 & 0.02725 & 0.01843 & 0.05809 \\
\hline 10 & RMW-10 & 0.00159 & 0.00427 & 0.01395 & 0.02223 & 0.00726 & 0.03366 & 0.04487 \\
\hline 11 & RMW-11 & 0.001176 & 0.00653 & 0.01838 & 0.2586 & 0.01931 & 0.00722 & 0.01719 \\
\hline 12 & RMW-12 & 0.00129 & 0.00343 & 0.00952 & 0.03138 & 0.02377 & 0.02024 & 0.10409 \\
\hline 13 & RMW-13 & 0.00199 & 0.00171 & 0.01569 & 0.03177 & 0.02299 & 0.02917 & 0.04330 \\
\hline 14 & RMW-14 & 0.001076 & 0.00221 & 0.01183 & 0.04520 & 0.02905 & 0.02671 & 0.01621 \\
\hline 15 & RMW-15 & 0.001636 & 0.00511 & 0.00932 & 0.002108 & 0.01877 & 0.02022 & 0.03798 \\
\hline 16 & RMW-16 & 0.001601 & 0.00396 & 0.01068 & 0.01418 & 0.03681 & 0.03757 & 0.03116 \\
\hline 17 & RMW-17 & 0.001967 & 0.00513 & 0.00977 & 0.1863 & 0.04545 & 0.02974 & 0.02739 \\
\hline 18 & RMW-18 & 0.001783 & 0.00523 & 0.01008 & 0.00183 & 0.04297 & 0.02099 & 0.07777 \\
\hline 19 & RMW-19 & 0.001500 & 0.00275 & 0.01325 & 0.0710 & 0.02320 & 0.02072 & 0.0474 \\
\hline 20 & RMW-20 & 0.0011 & 0.00477 & 0.01330 & 0.2056 & 0.00889 & 0.0109 & 0.0474 \\
\hline 21 & RMW-21 & 0.002527 & 0.00238 & 0.01497 & 0.1084 & 0.02511 & 0.03597 & 0.03568 \\
\hline 22 & RMW-22 & 0.002125 & 0.00538 & 0.01540 & 0.12183 & 0.02647 & 0.01215 & 0.0588 \\
\hline 23 & RMW-23 & 0.001467 & 0.0041 & 0.01538 & 0.1060 & 0.02460 & 0.02879 & 0.0588 \\
\hline 24 & RMW-24 & 0.001657 & 0.00402 & 0.01475 & 0.09593 & 0.02403 & 0.02503 & 0.02641 \\
\hline 25 & RMW-25 & 0.003109 & 0.00287 & 0.01348 & 0.07937 & 0.01703 & 0.02605 & 0.03956 \\
\hline 26 & RMW-26 & 0.002575 & 0.00516 & 0.01434 & 0.03414 & 0.01860 & 0.04132 & 0.02687 \\
\hline 27 & RMW-27 & 0.002301 & 0.00291 & 0.01326 & 0.17985 & 0.02052 & 0.02248 & 0.02888 \\
\hline 28 & RMW-28 & 0.00166 & 0.00319 & 0.01129 & 0.18292 & 0.02458 & 0.01896 & 0.01764 \\
\hline 29 & RMW-29 & 0.002506 & 0.00505 & 0.01158 & 0.10898 & 0.01925 & 0.01998 & 0.03106 \\
\hline 30 & RMW-30 & 0.00187 & 0.00464 & 0.01128 & 0.2052 & 0.0270 & 0.02109 & 0.0744 \\
\hline 31 & RMW-31 & 0.00224 & 0.00448 & 0.01389 & 0.12299 & 0.0281 & 0.03178 & 0.0392 \\
\hline
\end{tabular}




\begin{tabular}{lllllllll}
\hline SI. No & Sample no & Cd (ppm) & Co (ppm) & Cu (ppm) & Mn (ppm) & Ni (ppm) & Pb (ppm) & Zn (ppm) \\
\hline 32 & RMW-32 & 0.00098 & 0.00209 & 0.01103 & 0.15089 & 0.0233 & 0.02655 \\
33 & RMW-33 & 0.00157 & 0.00615 & 0.010119 & 0.0823 & 0.0238 & 0.0175 \\
34 & RMW-34 & 0.00228 & 0.00434 & 0.00969 & 0.1976 & 0.0283 & 0.02516 \\
35 & RMW-35 & 0.002398 & 0.00329 & 0.01055 & 0.1955 & 0.0267 & 0.01051 \\
36 & RMW-36 & 0.001512 & 0.00330 & 0.01306 & 0.36979 & 0.0212 & 0.0398 \\
\hline
\end{tabular}

Table 4. Chemical Analysis of water samples.

\begin{tabular}{|c|c|c|c|c|c|c|c|}
\hline Sl. No & Sample no & Al (ppm) & Ca (ppm) & $\mathrm{Fe}(\mathrm{ppm})$ & Mg (ppm) & $\mathrm{Na}(\mathrm{ppm})$ & K (ppm) \\
\hline 1 & RMW-1 & 0.42455 & 26.2127 & 1.3924 & 9.6399 & 19.2680 & 14.0639 \\
\hline 2 & RMW-2 & 46.2870 & 26.6653 & 3.9565 & 7.5385 & 17.5438 & 10.1691 \\
\hline 3 & RMW-3 & 1.6361 & 1.4346 & 0.1157 & 0.2831 & 0.9372 & 2.3932 \\
\hline 4 & RMW-4 & 2.3254 & 2.1030 & 0.1598 & 0.4073 & 1.3650 & 7.2613 \\
\hline 5 & RMW-5 & 2.7760 & 2.4870 & 0.1879 & 0.4701 & 0.9854 & 7.8560 \\
\hline 6 & RMW-6 & 2.5630 & 2.29698 & 0.1708 & 0.4543 & 1.2624 & 2.2894 \\
\hline 7 & RMW-7 & 2.18927 & 1.97678 & 0.1402 & 0.3850 & 1.2184 & 2.2409 \\
\hline 8 & RMW-8 & 2.1668 & 1.99539 & 0.1283 & 0.3651 & 1.2409 & 16.1534 \\
\hline 9 & RMW-9 & 1.5100 & 1.4447 & 0.0862 & 0.2466 & 0.8440 & 1.6350 \\
\hline 10 & RMW-10 & 1.550 & 1.3915 & 0.0878 & 0.2578 & 0.8627 & 4.1021 \\
\hline 11 & RMW-11 & 1.4308 & 1.3252 & 0.0763 & 0.2323 & 0.8275 & 1.2555 \\
\hline 12 & RMW-12 & 0.9910 & 0.8766 & 0.0541 & 0.1567 & 0.5225 & 5.64516 \\
\hline 13 & RMW-13 & 1.0088 & 0.8957 & 0.0551 & 0.1608 & 0.5797 & 6.42626 \\
\hline 14 & RMW-14 & 1.0451 & 0.9488 & 0.0545 & 0.1625 & 0.5453 & 0.7494 \\
\hline 15 & RMW-15 & 0.7564 & 0.72076 & 0.0426 & 0.1194 & 0.4510 & 2.4867 \\
\hline 16 & RMW-16 & 0.7229 & 0.67687 & 0.0378 & 0.1157 & 0.4178 & 0.75323 \\
\hline 17 & RMW-17 & 0.6778 & 0.62886 & 0.0353 & 0.10713 & 0.3501 & 0.8931 \\
\hline 18 & RMW-18 & 0.5557 & 0.4986 & 0.0279 & 0.0824 & 0.2549 & 0.3494 \\
\hline 19 & RMW-19 & 0.4707 & 0.59816 & 0.0235 & 0.0684 & 0.2234 & 1.9290 \\
\hline 20 & RMW-20 & 0.4508 & 0.68371 & 0.0211 & 0.0642 & 0.21417 & 4.5324 \\
\hline 21 & RMW-21 & 0.5374 & 0.5122 & 0.0260 & 0.863 & 0.2565 & 5.970 \\
\hline 22 & RMW-22 & 0.4206 & 0.6246 & 0.0198 & 0.0620 & 0.1942 & 5.2580 \\
\hline 23 & RMW-23 & 0.5789 & 0.5635 & 0.0303 & 0.11006 & 0.2817 & 6.705 \\
\hline 24 & RMW-24 & 2.2229 & 25.7398 & 0.6011 & 8.6409 & 20.9964 & 5.9465 \\
\hline 25 & RMW-25 & 0.8700 & 25.9851 & 0.7396 & 8.7592 & 21.8164 & 6.3779 \\
\hline 26 & RMW-26 & 0.4288 & 24.0763 & 0.2754 & 8.1267 & 23.3166 & 7.3639 \\
\hline 27 & RMW-27 & 0.0518 & 7.2724 & 2.2154 & 2.7361 & 14.1131 & 0.8698 \\
\hline 28 & RMW-28 & 0.05266 & 8.4242 & 2.2913 & 3.8452 & 17.3691 & 1.3034 \\
\hline 29 & RMW-29 & 0.51669 & 25.2039 & 1.2053 & 9.429 & 19.0092 & 7.451 \\
\hline 30 & RMW-30 & 0.06167 & 11.1777 & 0.1463 & 4.6642 & 15.2152 & 3.0522 \\
\hline 31 & RMW-31 & 0.3362 & 15.2887 & 1.18763 & 9.49732 & 21.0992 & 6.8966 \\
\hline 32 & RMW-32 & 0.1385 & 14.2725 & 2.40421 & 6.4139 & 19.3978 & 2.625 \\
\hline 33 & RMW-33 & 0.2904 & 12.1848 & 1.01344 & 9.3415 & 19.9985 & 7.945 \\
\hline 34 & RMW-34 & 0.06533 & 13.0987 & 4.8102 & 6.1034 & 21.8866 & 1.850 \\
\hline 35 & RMW-35 & 0.16737 & 11.9877 & 0.75933 & 7.1438 & 22.098 & 1.0532 \\
\hline 36 & RMW-36 & 0.1292 & 8.6799 & 1.3399 & 4.73924 & 17.0689 & 2.5723 \\
\hline
\end{tabular}

wind is calm.

\section{Meteorological Condition}

Meteorological information relevant to the Barapukuria site is available from weather stations at Dinajpur and Rangpur. These stations are about $30 \mathrm{~km}$ west and east respectively from the project site. The average annual precipitation in the area is 1,800 to $2,000 \mathrm{~mm}$ of which $85 \%$ comes from May to September. Heavy rainfall for $24 \mathrm{~h}$ or more are recorded which in succession causes 1 to $1.5 \mathrm{~m}$ depth flood in the low-lying areas of the vicinity. The relative humidity is above $80 \%$ at daytime and $90 \%$ at night time. The maximum temperature is $38^{\circ} \mathrm{C}$ in June and the lowest is $4^{\circ} \mathrm{C}$ in December. The available data on wind directions and speeds indicate that, the wind blows predominantly from East to West $(40 \%)$, West to East $(25 \%)$ and from North-East $(18 \%)$. The wind speed rarely exceeds $8 \mathrm{~m} / \mathrm{s}$ and mostly the

\section{Results and Discussions}

The International Accountability Project reports that mining operations at Barapukuria have destroyed roughly 300 acres of land, impacting about 2,500 people in seven villages, as land subsidence of over one meter in depth has destroyed crops and lands and damaged homes. People in 15 villages have also reportedly lost their access to water, as huge quantities of water pumped out for the Barapukuria mine caused a rapid drop in water levels. [9] The environmental impact of Barapukuria Coal Mining Company Ltd. and Barapukuria Thermal Power Plant Ltd. is generally observed in two ways. Physical effects are the physical changes in the vegetation, soil, water and structure around the area. The chemical effect is a change in the amount of chemical elements present in the soil and water in the area. 
Physical effects show in a short time. Chemical effects are usually seen after a long time.

The colour of the water samples of coal leached drainage water appeared blackish and the agricultural land water samples appeared slightly blackish. Therefore, the water was unsuitable not only for aquaculture but also for domestic, industrial or agricultural purposes. However, the groundwater and surface water were colorless. The temperature recorded in water samples beside the mine drainage was $40^{\circ} \mathrm{C}$ whereas the temperature of agricultural land water, surface and groundwater was 32,25 and $22^{\circ} \mathrm{C}$, respectively (Table 2).

The $\mathrm{pH}$ values measured in Barapukuria coal mine industry showed that all of the samples have $\mathrm{pH}$ values of greater than 7, which is slightly alkaline whereas the standard value of $\mathrm{pH}$ was 6 to 9 (ECR 1997) [10]. The $\mathrm{pH}$ value of mine drainaged water was recorded 7.55 whereas the $\mathrm{pH}$ values of agricultural land water, groundwater and surface water were $7.4,7.45$ and 7.52, respectively (Table 1), which reflects its suitability for aquatic life and for all types of water uses.

The EC and TDS at different sampling points were ranged from $204-370 \mu \mathrm{S} / \mathrm{cm}$ and $104-198 \mathrm{mg} / \mathrm{l}$, respectively. The highest TDS $(198 \mathrm{mg} / \mathrm{l})$ and $\mathrm{EC}(370 \mu \mathrm{S} / \mathrm{cm})$ were founding surface water and in mine drainaged water, respectively (Table 2). Water that contains less than $500 \mathrm{ppm}$ of dissolved solid is generally satisfactory for the domestic use and other industrial purposes and water containing more than 1000 ppm of dissolved solids usually contains minerals that give it a distinctive taste or make it unsuitable for human 68 defined the standard value of TDS for the inland surface water as $2100 \mathrm{mg} / \mathrm{l}$. As the total concentration of dissolved solids in water is a general indication of its suitability for any particular purpose, the result of the study concluded that the water at the downstream of the river is considered as suitable for fish culture and other purposes.

The mining authority release remaining waste water into the mining drain. The local people used this water in agriculture. Last December 2017 Barapukuria Thermal Power Station has already started using unused water from Barapukuria Coal Mine in this thermal power plant. The Barapukuria thermal power plant discharges unused water into the nearby Tilai river through a drain. The temperature of the water is about $46^{\circ} \mathrm{C}$. The high temperature of abandoned water is affecting the biodiversity.

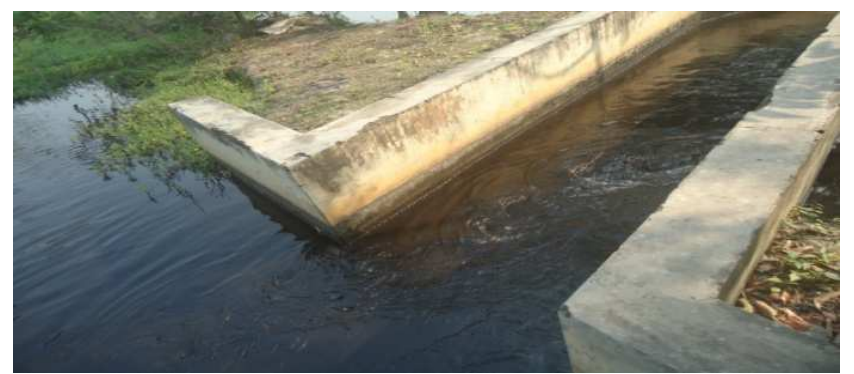

Figure 2. The end of the drain of the abandoned water flow of the Barapukuria coal mine.

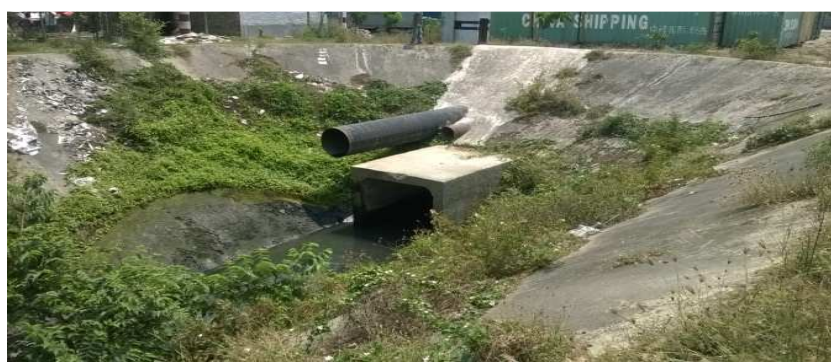

Figure 3. Drainage water used by thermal power plants.

Most of area of Bouddhanathpur (Jigagari) and Gopalpur (Maupukur) near Barapukuria Coal Mining Company Ltd. have been subside, creating a reservoir about 5-6 m deep.

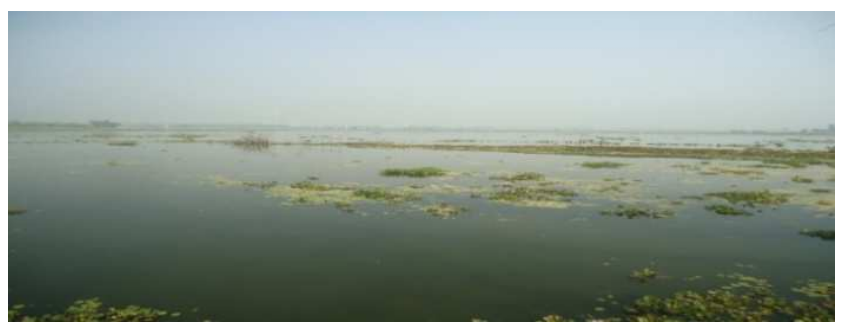

Figure 4. Subside reservoirs created as a result of coal extraction.

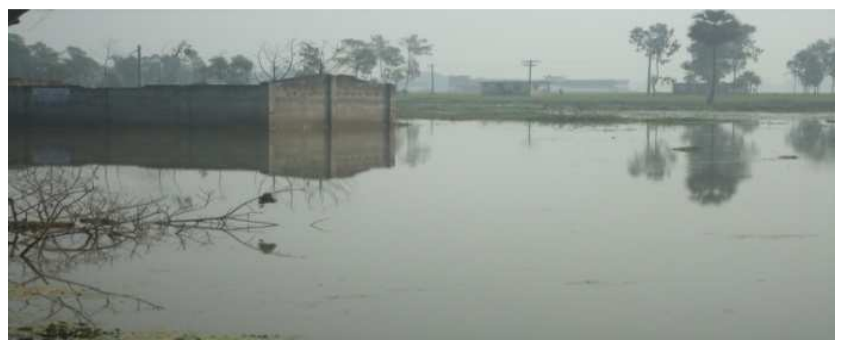

Figure 5. Reservoir created in the new area of Subside as a result of coal extraction.

\section{Conclusion}

The study showed the overall scenario regarding the concentration of different water quality parameters resulted from the Barapukuria coal mine activity and their suitability for aquatic life as well as for irrigation. The results of the study concluded that the water in the coal mine industrial area were found quietly contaminated, which can deteriorate the aquatic life and agriculture. The study showed that all the water quality parameters of mining area i.e. temperature, $\mathrm{pH}, \mathrm{EC}, \mathrm{TDS}, \mathrm{DO}$ were in standard levels. The average concentration of $\mathrm{Cu}, \mathrm{Fe}$ and $\mathrm{Zn}$ content in the water samples was below the standard level. The study identified that the $\mathrm{pH}$ of the water samples were in permissible level and thus suitable for agriculture. Although the concentration of heavy metals in the water samples was in permissible limits, however the study depicted that high levels of these heavy metals from the coal mine can pose a serious threat on the environment within a short period of time.

\section{Acknowledgements}

The authors wishes to express their heartiest gratitude, 
warmest thanks to Mr. Resad Mohammad Ekram Ali, Director General of Geological Survey of Bangladesh (GSB) for giving the opportunity to get this work done.

The authors are indebted to Mr. Ahmed Ataul Muneem, Director (Chemistry) \& Branch Chief of Analytical Chemistry Branch of GSB for his invaluable guidance and administrative cooperation throughout this work. The authors are also thankful to all officers and staffs of the Analytical Chemistry Branch of GSB for their necessary help and assistance during field program as well in the laboratory work.

The authors are sincerely thanks to Mr. Hossain Mohammad Arifin, Assistant Director (Geology) of GSB for his support to prepare a location map of this work.

Above all the authors are very much thankful to all officers \& staff of other Branches, specially administration, transport, accounts, etc also involve in different way in successfully completion of this programme.

\section{References}

[1] Ashton, P. J. 1999. Using environmental impact assessment to determine the consequences of mining activities and highlights the costs of sustainable development. In: Proceedings of the Conference of the Environmental Management System in Mining, 11-13 October, Kemptonpark, South Africa, p. 12.

[2] Ashton, P. J., D. Love, H. Mahachi, P. H. G. M. Dirks. 2001. An overview of the impact of Miningand mineral processing operations on water resources and water quality in the Zambezi, Limpopo and Olifants catchments in Southern Africa. Contract Report to the Mining, ineralsand Sustainable Development (Southern Africa) project by, CRIS environment. Tiwary, R. K. 2001. Environmental Impact of Coal Mining on Water Regime and Its Management. Water, Air, and Soil Pollution. 132: 185-199.

[3] B. Tutmez, B. Hozatli, and A. K. Cengiz, An Overview of Turkish Lignite Qualities by Logistic Analysis, Journal of Coal Science, \& Engineering, 19, 2013, 113-118.
[4] E. Morrice and Colagiuri, Coal Mining, Social Injustice and Health: A Universal Conflict of Power and Priority", Health \& Place, 19, 2013, 74-79.

[5] V. Saini, R. P. Gupta, M. K. Arora, Environmental Impact Studies in Coalfields in India: A Case Study from Jharia Coalfield, Renewable and Sustainable Energy Review, 53, 2016, 1222-1239.

[6] IIED, Coal Case Study, 66, 2002, 1-9.

[7] Lindsay, W. L., 1979. Chemical equilibria in soils. John Wiley and Sons. New York.

[8] S. A. M. Din, N. N. N. Yahya, A. Abdullah, Fine Particulates Matter (PM2.5 from Coal-Fired Power Plant in Manjung and its Health. Impacts", Procedia-Socio and Behavioral. Science, 85. 2013, 92-99.

[9] KateHoshour, "Massive protest against Phulbari\&Barapukuria coal mines in Bangladesh" International Accountability Project, March 4, 2011.

[10] ECR (Environment Conservation Rules). 1997. Government of the Republic of Bangladesh. Ministry of Environment and Forest.

[11] Appelo, C. A. J. and D. Postma. 1993. Geochemistry, ground water and pollution. A. A. Balkema Publisher, USA.

[12] Bakr AM, Rahman QMA, Islam MM, Islam MK, Uddin MN, Resan SA, Haider MJ, Islam MS, Ali MW, Chowdhury M, Mannan KH, Anam ANMH (1996) Geology and coal deposit of Barapukuria basin, Dinajpur district, Bangladesh. Records of the Geological Survey of Bangladesh 8 (1): 36 .

[13] Energy Information Administration Reports 2005. The official energy statistics of the U.S. Government.

[14] Rapid Analysis of Silicate, Carbonate, and Phosphate RocksRevised Edition. Geological Survey of Bulletin 1401.

[15] Quality criteria for water. U.S. Environmental protection agency, Washington, D. C. 20460.

[16] Groundwater Geochemistry fundamentals and Applications to Contamination-William J. Deutsch. Lewis Publishers. 